Dev Neurosci 2017;39:171-181

DOI: $10.1159 / 000460815$
Received: September 15, 2016

Accepted after revision: February 8, 2017 Published online: April 22, 2017

\title{
Unbiased Quantification of Subplate Neuron Loss following Neonatal Hypoxia-Ischemia in a Rat Model
}

\author{
Alexandra Mikhailova Naveena Sunkara Patrick S. McQuillen \\ Department of Pediatrics, School of Medicine, University of California, San Francisco, San Francisco, CA, USA
}

\section{Keywords}

Optical Fractionator · Cortical layer-specific marker · Brain injury · Premature newborn

\begin{abstract}
Background: Cellular targets of neonatal hypoxia-ischemia $(\mathrm{HI})$ include both oligodendrocyte and neuronal lineages with differences in the patterns of vulnerable cells depending upon the developmental stage at which the injury occurs. Injury to the developing white matter is a characteristic feature of human preterm brain injury. Data are accumulating, however, for neuronal injury in the developing cerebral cortex. In the most widely used rodent model of preterm $\mathrm{HI}$ brain injury, conflicting data have been reported regarding the sensitivity of subplate neurons to early neonatal $\mathrm{HI}$, with some reports of selective vulnerability and others that find no increased loss of subplate neurons in comparison with other cortical layers. Methods used to identify subplate neurons and quantify their numbers vary across studies. Objective: To use recently developed cortical layer-specific markers quantified with definitive stereologic methods to determine the magnitude and specificity of subplate neuron cell loss following neonatal $\mathrm{HI}$ in a rodent model. Methods: Postnatal day 2 (P2) rats underwent right common carotid artery coagulation followed by $2-3 \mathrm{~h}$ of hypoxia (5.6\% oxygen). Categorically moderately injured brains were stained
\end{abstract}

with subplate and cortical layer III-V markers (Complexin3 and Foxp1, respectively) at P8 and P21 (Foxp1 only). An Optical Fractionator was used to quantify subplate and middle/ lower cortical neuronal numbers and these were compared across groups (naive control, hypoxia hemisphere, and $\mathrm{HI}$ hemisphere). Results: Following $\mathrm{HI}$ at P2 in rats, the total Complexin3-expressing subplate neuron number decreases significantly in the $\mathrm{HI}$ hemisphere compared with naive controls or hypoxia alone (HI vs. control $26,747 \pm 7,952$ vs. $35,468 \pm 8,029, p=0.04 ; \mathrm{HI}$ vs. hypoxia, $26,747 \pm 7,952$ vs. $40,439 \pm 7,363, p=0.003)$. In contrast, the total Foxp1-expressing layer III-V cell number did not differ across the 3 conditions at P8 (HI vs. control 1,195,085 $\pm 436,609$ vs. $1,234,640 \pm 178,540, p=0.19 ; \mathrm{HI}$ vs. hypoxia, 1,195,085 \pm 436,609 vs. $1,289,195 \pm 468,941, p=0.35$ ) and at $\mathrm{P} 21$ (HI vs. control $1,265,190 \pm 48,089$ vs. $1,195,632 \pm 26,912, p=0.19$; HI vs. hypoxia, $1,265,190 \pm 48,089$ vs. $1,309,563 \pm 41,669$, $p=0.49$ ). Conclusions: There is significant biological variability inherent in both the subplate neuron cell number and the pattern and severity of cortical injury following $\mathrm{HI}$ at P2 in rats. Despite this variability, the subplate neuron cell number is lower following $\mathrm{P} 2 \mathrm{HI}$ in animals with mild or moderate cortical injury, whereas the middle-to-lower-layer cortical neuronal number is unchanged. In more severe cases, neurons are lost from the lower cortical layers, suggesting a relative vulnerability of subplate neurons.

(c) 2017 S. Karger AG, Basel

\section{KARGER}

(c) 2017 S. Karger AG, Basel 


\section{Introduction}

Brain injury associated with premature birth is a major and increasing worldwide problem resulting in lifelong disability with substantial social and economic burdens $[1,2]$. The incidence of premature birth is rising along with improved survival even at extreme limits of viability. Rates of cognitive and behavioral disabilities rise with decreasing gestational age at birth [3]. Together, these trends result in a growing population of children with substantial neurodevelopmental disability.

Neurobiological substrates of motor disabilities following neonatal brain injury (e.g., cerebral palsy) have been attributed to specific neuroanatomical injuries. Spastic diplegia, for example, is thought to result from injury to myelinated pyramidal tract motor axons as they pass close by margins of the lateral ventricles coinciding with the common location for cystic white-matter injury (periventricular leukomalacia) [4]. The neurobiological substrates for cognitive and behavioral impairments are not as well understood but are likely to emerge from more widespread injuries involving multiple cortical areas.

Animal models that faithfully reproduce the brain injuries of human preterm infants are needed to gain insight into cellular and developmental mechanisms that result in the dysfunction of neural systems. Hypoxia-ischemia (HI) and inflammation are the most frequent causes of neonatal brain injury and can result in lifelong motor and cognitive disability [5]. Thus HI, with or without additional inflammation, in rodents, is the most common model of neonatal brain injury. Using this model immediately after birth combined with birth-dating methods, we reported selective vulnerability of subplate neurons following HI at postnatal day 2 (P2) [6]. The injury was deemed selective based upon a decrease in subplate neurons even in mild injuries, where no histologic injury could be noted in the Nissl-stained cortex. Severely injured cases were noted to have a loss of lower-layer neurons $[6,7]$.

This report was among the first to note direct neuronal injury following neonatal $\mathrm{HI}$ in addition to the more widely studied injury to preoligodendrocytes [8] and the activation of astrocytes [9] and microglia [10]. Subplate neurons play a critical role at many stages of cortical development before undergoing programmed cell death [11-13]. Subplate neurons pioneer projections from the cortex to the thalamus and, in turn, receive the first thalamic inputs [14-16]. Subplate neurons send a projection into layer IV of the neocortex, and thus form a transient developmental circuit through which they influence early cortical activity [17]. Subplate neurons play a role in the maturation of both excitatory and inhibitory cortical synapses $[7,18,19]$. Premature removal of subplate neurons alters specific cortical activity patterns and reduces the capacity for both early somatosensory barrel plasticity and later ocular dominance plasticity [7, 20-22]. Thus, the involvement of subplate neurons in a model of early brain injury offered a potential mechanism for the widespread dysfunction of cortical circuits observed following very preterm human birth.

Until recently, subplate neurons were identified by their characteristic morphology (inverted pyramidal), their location at the base of the neocortex (e.g., layer VIb in the rat), and by early birth dates [23]. However, as with neurons in all neocortical layers, subplate neurons are a pleomorphic population. Even prior to the development of cortical layer-specific markers, it was known that subplate neurons consisted of both excitatory and inhibitory populations with differing projection patterns [17]. With the advent of large-scale projects to survey regional and developmental gene expression (e.g., the Allen Institute Brain Atlas Project), new markers have been described for most cortical layers, including the subplate [24]. Using these and other markers, further diversity has been demonstrated for neurons in the subplate layer [25].

Given the conceptual importance of subplate neuron injury for neurodevelopmental impairments following human preterm birth and to more fully characterize the specific vulnerability of subplate neurons in this model, we used cortical layer-specific markers and definitive stereological methods to quantify subplate neuron number following neonatal $\mathrm{HI}$.

\section{Materials and Methods}

\section{Animals}

Timed-pregnant Long-Evans rats of both genders (Simonsen) were allowed food and water ad libitum. All animal research was approved by the University of California San Francisco Committee on Animal Research and performed in accordance with standards of humane care set forth in the Policy on Humane Care and Use of Laboratory Animals.

\section{Hypoxia-Ischemia}

The procedure was described previously [7,22]. The manipulation was performed at $\mathrm{P} 2($ day of birth $=\mathrm{P} 0)$. To produce ischemia, pups were individually anesthetized with isoflurane. A midline incision was made in the neck; the right common carotid artery was dissected from the jugular vein and coagulated with a bipolar coagulator. The animals were returned to the dam for 1-2 h to recover from anesthesia and feed. Subsequently, pups receiving hypoxia were placed in $5.6 \%$ oxygen in temperature-controlled 
Table 1. Stereology study parameters

\begin{tabular}{|c|c|c|c|}
\hline Parameters & At P8, Cplx3 & At P8, Foxp1 & At P21, Foxp1 \\
\hline Observed group mean, $n^{1}$ & $35,457(26,747)$ & $1,234,640(1,195,085)$ & $1,195,632(1,265,190)$ \\
\hline Observed coefficient of variation of group mean, $\mathrm{OCV}^{1}$ & $0.23(0.30)$ & $0.14(0.37)$ & $0.023(0.038)$ \\
\hline Observed coefficient of variation of estimator, $\mathrm{CE}^{1}$ & $0.08(0.10)$ & $0.08(0.08)$ & $0.05(0.05)$ \\
\hline Section sampling fraction, $s s f$ & $1 / 12$ & $1 / 12$ & $1 / 12$ \\
\hline Fraction of area of section sampled, asf & 0.1024 & 0.0028 & 0.0064 \\
\hline Dissector volume, $h \times A_{\text {frame }}$ & 16,000 & 4,000 & 16,000 \\
\hline Average number of objects counted in each individual, $\Sigma Q^{-}$ & 208 & 204 & 366 \\
\hline Thickness of mounted section, $t$ & $16.3 \mu \mathrm{m}$ & $15 \mu \mathrm{m}$ & $17.4 \mu \mathrm{m}$ \\
\hline Height of dissector, $h$ & $10 \mu \mathrm{m}$ & $10 \mu \mathrm{m}$ & $10 \mu \mathrm{m}$ \\
\hline Guard zone & $1 \mu \mathrm{m}$ & $1 \mu \mathrm{m}$ & $1 \mu \mathrm{m}$ \\
\hline Smoothness factor, $m$ & 1 & 1 & 1 \\
\hline
\end{tabular}

${ }^{1}$ Values denote: control hemisphere (HI injured hemisphere).

chambers maintained between $34-35^{\circ} \mathrm{C}$ for $2-3 \mathrm{~h}$. One pup from each litter was monitored with a skin surface temperature probe and the chamber temperature was adjusted to maintain skin temperature $\left(35.5-37^{\circ} \mathrm{C}\right)$. Hypoxia was continued for a maximum of $3.5 \mathrm{~h}$ or terminated for any individual pup at any signs of agonal respirations or any persistent clonic or tonic-clonic movements. At P8 or P21, the rat pups were sacrificed with $100 \mathrm{mg} / \mathrm{kg}$ pentobarbital given by intraperitoneal injection.

\section{Immunohistochemistry}

Animals were perfused transcardially with $0.1 \mathrm{M}$ phosphate buffer followed by cold $4 \%$ paraformaldehyde in $0.1 \mathrm{M}$ phosphate buffer. Perfused brains were postfixed for $6 \mathrm{~h}$ and then cryoprotected in $25 \%$ sucrose in $0.1 \mathrm{M}$ phosphate buffer overnight prior to sectioning on a sliding microtome. Eighty-micrometer sections were quenched with $3 \%$ hydrogen peroxide, washed, and blocked (5\% donkey serum, 10\% BSA, 5\% fish skin gelatin, and $0.1 \%$ Triton $\mathrm{X}-100$ in $0.1 \mathrm{M}$ phosphate-buffered saline). Primary antibody dilutions used were 1:5,000 for anti-Complexin3 (rabbit polyclonal, 122 302; Synaptic Systems) and 1:50,000 for anti-Foxp1 (mouse monoclonal, ab32010; AbCam). Primary antibody was applied overnight at $4^{\circ} \mathrm{C}$. Secondary antibody 1:500 (biotinylated donkey anti-mouse or biotinylated donkey anti-rabbit; Jackson ImmunoResearch) was applied for $1 \mathrm{~h}$ at room temperature. The sections were washed and incubated with avidin-biotinylated complex (ABC) using the Vectastain Elite kit (Vector Laboratories, Burlingame, CA, USA) for $2 \mathrm{~h}$. Lastly, the sections were washed and developed in a solution of 3,3'-diaminobenzidine (Sigma, St. Louis, MO, USA) and nickel sulfate for $3 \mathrm{~min}$. For fluorescent labeling, the same procedure was followed, omitting hydrogen peroxide incubation. Following primary incubation and washing, sections were incubated with secondary antibodies 1:500 donkey anti-rabbit (AlexaFluor488; Jackson) or 1:500 donkey anti-goat (AlexaFluor594; Life Technologies).

Stereological Study of Subplate Neuron Loss after Neonatal HI

\section{Quantification of Cell Numbers}

We performed a formal stereology study that followed a systematic random-sampling scheme: sections were selected systematically (every 12th) from the front to the back of the brain, and the probing of sections was randomized such that all parts of the region of interest had an equal probability of being sampled. The subplate zone was identified (as described previously [6]) by the cytoarchitectonic features of the neocortex [23]. In the radial domain, the subplate (layer VIb) was localized at the base of the cortical plate, immediately below the layer VI neurons, and contained characteristic inverted pyramidal neurons. The borders in the coronal domain extended to the cingulate cortex in the medial direction and the entorhinal cortex laterally. The rostral and caudal boundaries were determined empirically, to allow for at least 1 section at each end of the brain to be discarded. Rostrally, the first sections were omitted if no corpus callosum was present. Caudally, sections without cingulate cortex were omitted. The layers (III-V) were determined radially with Foxp 1 heavily stained cells in the characteristic 6-layered neocortex, with borders extending into the cingulate cortex in the medial direction and the entorhinal cortex laterally. The same rostral/caudal boundaries were used for subplate and cortical-layer markers. Cell populations were estimated using the Optical Fractionator probe [26] in Stereo Investigator (MBF Bioscience, Williston, VT, USA). We have reported the estimated population (number-weighted section thickness) for all cell counts. The parameters of the Optical Fractionator study are given in Table 1. Four to six sections were analyzed per animal at P8, and 7-8 sections at P21. Individual cells were counted using standard stereological methods with a $\times 63$ oil immersion lens on a Zeiss Axioscope II epifluorescent microscope. Adequate sampling was verified with coefficients of error (CE, e.g., Gundersen, $m=1)<0.1$, and were later analyzed for biological and stereological variability (see Results). 
Fig. 1. Subplate markers Nurr1 and CTGF colabel some cells with Cplx3. P8 rat control brain stained with subplate markers Complexin3 (green) and Nurr1 (red) (a), or Complexin3 (green) and CTGF (red) (b) shows cells positive for both or either markers. Scale bar, $100 \mu \mathrm{m}$. $\mathbf{a}^{\prime}$, b' Corresponding panels show a close-up of singlepositive and double-positive cells. Scale bar, $10 \mu \mathrm{m}$.
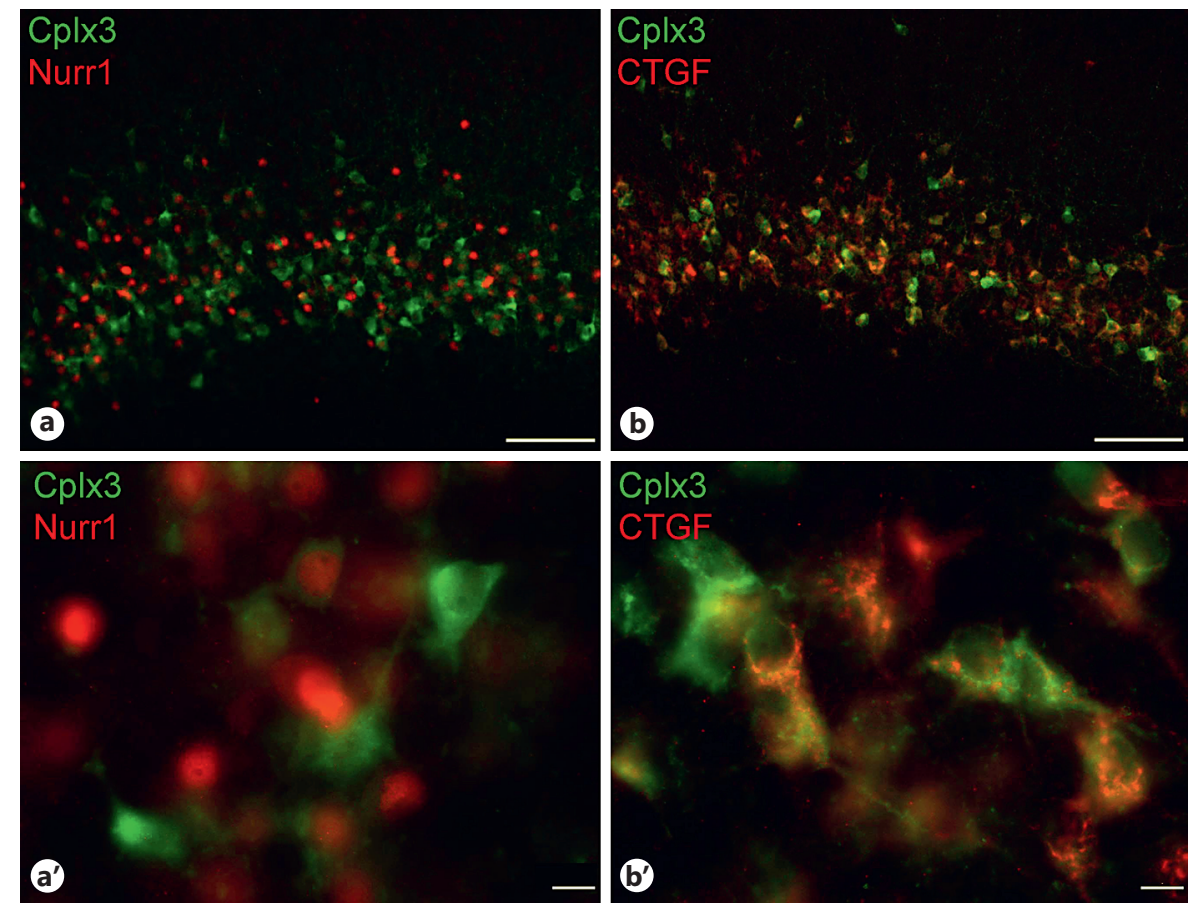

\section{Statistics}

Data were examined graphically with scatter plots to evaluate distribution and were summarized as mean \pm SD. Sample population difference testing was performed with the unpaired, 2-sided Student $t$ test. Association was tested by linear regression. Power calculations were performed using sample size, effect size, and SD as described [27]. Biological variability was estimated using the coefficient of variance (CV, mean/SD) and CE (described in [28]).

\section{Results}

\section{Determining a Marker for Stereology}

For a marker to be compatible with stereological counting procedures, it must localize to a discernible point or profile to determine that it falls within the boundaries of the counting frame/dissector. To select a subplate marker suitable for stereological counting, we examined 3 previously used options [25, 29]: Complexin3, Nurr1, and CTGF. We performed fluorescent stains with all 3 (Fig. 1). These 3 markers label overlapping but not identical subplate populations. Complexin3 may colabel with Nurr1 or CTGF, but single positive cells for each marker are noted as well. Nurrl staining is nuclear and technically ideal for counting (Fig. 1a'). However, Nurr1-labeled cortical cells occur outside of the subplate, especially near the entorhinal cortex. CTGF staining is cytoplasmic and punctate with irregular profiles and is not ideal for top-counting (Fig. 1b'). Complexin3 is cytoplasmic, outlining the cell nucleus, and, importantly, is the most robust with peroxidase-DAB staining, labeling only the cells in the subplate but not elsewhere in the cortex.

To select layer-specific markers suitable for the stereological quantification of cortical layers, we considered: Er81 (layer V), Tbr1 (layer VI), Foxp2 (layer VI), Ctip2 (layer V-VI), and Foxp1 (layers III-V). Er81 selectively labels a subpopulation of layer $\mathrm{V}$ cells, and some in layer III-IV (Yamamoto 2006). While Er81 worked robustly for Okusa et al. [29], in our experience, a commercially available Er81 antibody (sc-28681, Santa Cruz Biotech) was not layer-specific. Foxp1 was chosen because of its clean nuclear stain with a clear layer specificity that included layer V.

\section{Histological Analysis of Moderate HI Injury at P8}

The Vannucci procedure [30], unilateral ligation of the common carotid artery followed by bilateral hypoxia, produces injury in the range of mild to severe, dependent upon a variety of factors including age, animal strain, and temperature [31]. At P2, we arbitrarily defined injury categories from mild (minimal to no histologic changes in the neocortex and mild or no asymmetry of lateral ventricles in the coronal plane), moderate (clear asymmetry of lateral ventricles with thinning or patchy dropout of layer $\mathrm{VIb} /$ subplate), and severe (widespread loss 

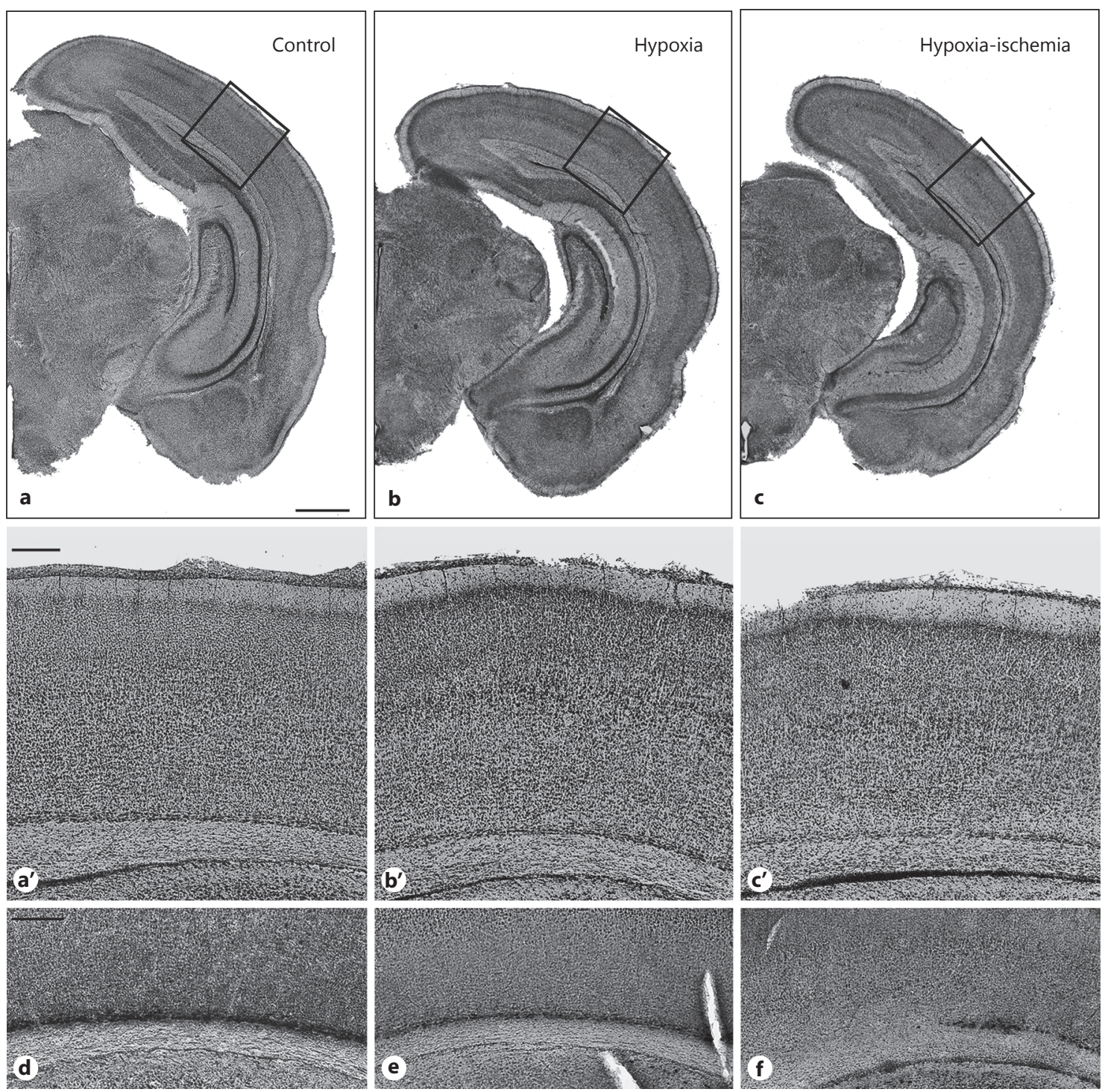

Fig. 2. Histological assessment of moderate cortical injury following hypoxia-ischemia in a $\mathrm{P} 8$ rat. Cresyl violet-stained coronal sections of the 3 examined conditions: naive control (a), hypoxia (contralateral to injury, b), hypoxia-ischemia (ipsilateral to injury, c). Scale bar, $1 \mathrm{~mm}$. $\mathbf{a}^{\prime}-\mathbf{c}^{\prime}$ Corresponding panels show a close-up of the areas indicated by black frames. Scale bar, $200 \mu \mathrm{m}$. Comparable regions stained for Complexin3 with DAB show a normal subplate (d), a thinning subplate in hypoxia (e), and patches of subplate cell loss in hypoxia-ischemia (f). Scale bar, $200 \mu \mathrm{m}$. of layer $\mathrm{VIb} /$ subplate and/or laminar or columnar loss of cells in layer VIa) [7]. Severe cases often have cyst formation laterally with more extensive loss of the cortical layers. Other investigators have reported similar patterns, although categorical definitions vary [32-34]. For continuous measures of injury severity, we employed cortical thickness measured from the pial to the ventricular surface over the primary visual cortex adjacent to the dentate gyrus at P30 [7]. More recently, we measured infarct volumes with the Cavalieri method [22]. Eight lit- ters ( 4 in the early cohort and 4 in the late cohort) were used for the present experiments. There were 85 pups in total and both genders were used; 12 were naive littermate controls. In total, 72 pups received $\mathrm{HI}$ at $\mathrm{P} 2$, with a procedural mortality of 12 (16\%). Cortical injury was identified on coronal sections stained with Cresyl violet, and was categorized based on the above criteria. Out of 60 of animals surviving HI, 43 developed mild injury, 15 had moderate injury, and 2 had severe injury. Histological changes including hemispheric asymmetry in the cor- 
Fig. 3. Regions of interest and counting frames for stereological quantification in a $\mathrm{P} 8$ rat. DAB staining with subplate marker, Complexin3 (a) and layer III-V marker, Foxp1 (b). Contours in red indicate regions of interest used for analysis. Scale bar, $1 \mathrm{~mm}$. Examples of a Complexin $3+$ cell $(\mathbf{c}$, counting frame: $40 \times 40 \mu \mathrm{m})$ and Foxp1+ cell $(\mathbf{d}$, counting frame: $20 \times 20 \mu \mathrm{m})$, counted using the Optical Fractionator probe.

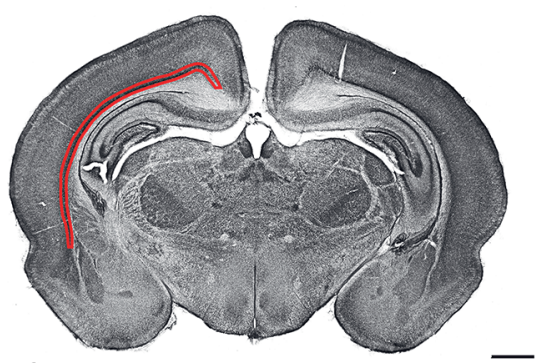

a

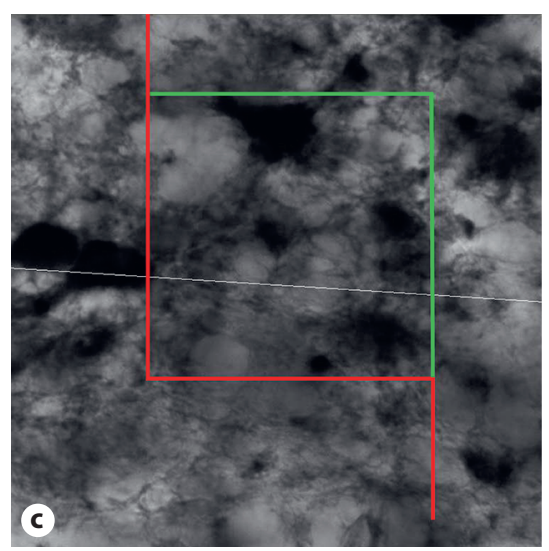

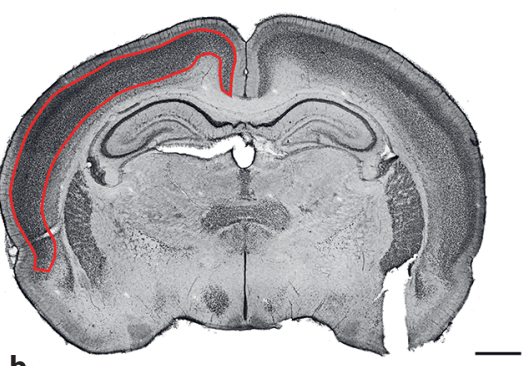

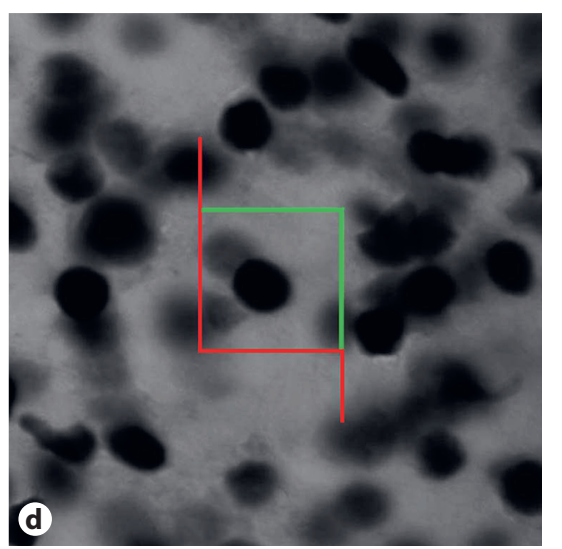

onal plane and thinning of layer VIb following $\mathrm{HI}$ are shown in Figure 2. At P8, hemispheric volumes ranged from 148 to $183 \mathrm{~mm}^{3}$ in the hypoxia-alone hemispheres and 124 to $183 \mathrm{~mm}^{3}$ in the HI-exposed hemispheres. Infarct volumes (hypoxia hemisphere - HI hemisphere)/ hypoxia hemisphere $\times 100$ ) ranged from -2 to $15 \%$. At P21, hemispheric volumes ranged from 220 to $324 \mathrm{~mm}^{3}$ in the hypoxia-alone hemispheres and 217 to $315 \mathrm{~mm}^{3}$ in the HI-exposed hemispheres. Infarct volumes ranged from 0 to $13 \%$.

\section{Significant Reduction in Subplate Cell Population in HI Injury Group}

Sections were selected using systematic random sampling and the Optical Fractionator method was used to quantify cell numbers [26]. For this procedure, contours were drawn using clear anatomical boundaries, and were guided by layer-specific marker immunohistochemistry (summarized in Methods). The counting and sampling of grid dimensions was determined with pilot studies (summarized in Table 1). Low magnification examples of the boundaries for both Complexin 3 and Foxp 1 are shown in Figure 3a, b. Individual cells were counted following stan- dard stereological procedures, and examples of the counted cells are shown in Figure 3c, d.

The total number of Complexin3-expressing cells was counted in the hypoxia and HI hemispheres of pups $(n=$ 8 ) with moderate brain injury and compared to naive controls $(n=5)$. Hemispheric counts are summarized in Figure 4a. Naive control animals had 35,456 $\pm 8,029$ cells per hemisphere at P8. Hemispheres exposed to hypoxia alone had 40,439 $\pm 7,363$ cells per hemisphere. We found a significant decrease in cell numbers in the HI group, i.e., $26,747 \pm 7,952$, compared to the hypoxia and naive control hemispheres ( $p=0.003$ and $p=0.004$, respectively). Hemispheric ratios (right/left hemisphere) are presented to account for interanimal variability in Complexin 3 and Foxp1 cell numbers (Fig. 4c).

\section{HI Injury Does Not Significantly Reduce the Number of Cells in Cortical Layers III-IV at P8 or P21}

To assess whether cell populations in the middle-tolower cortical layers were affected by $\mathrm{HI}$ insult at P8, we counted the number of Foxp1+ cells in the ipsilateral and contralateral hemispheres of moderately injured HI brains $(n=8)$ and compared these with naive controls 
Fig. 4. Quantification of Complexin 3 and Foxp1 across injury groups at P8. The Complexin3+ subplate population in the hypoxia-ischemia (HI) group is significantly decreased compared to that in the control and hypoxia group (a, $p=0.0177$ and $p=0.0015)$. $\mathbf{b}$ Foxp $1+$ cell numbers are not significantly different between injury groups. c Hemispheric (right/left, R/L) ratios of Complexin 3 and Foxp1 counts show a greater variability in the injured groups than in controls. Circles indicate controls, squares indicate HI. d Complexin3 cell counts in the HI hemisphere are negatively correlated with infarct volume, as defined by (infarct volume $=[$ hypoxia $-\mathrm{HI}] /$ hypoxia). $r^{2}=0.82,{ }^{*} p=0.0017$.

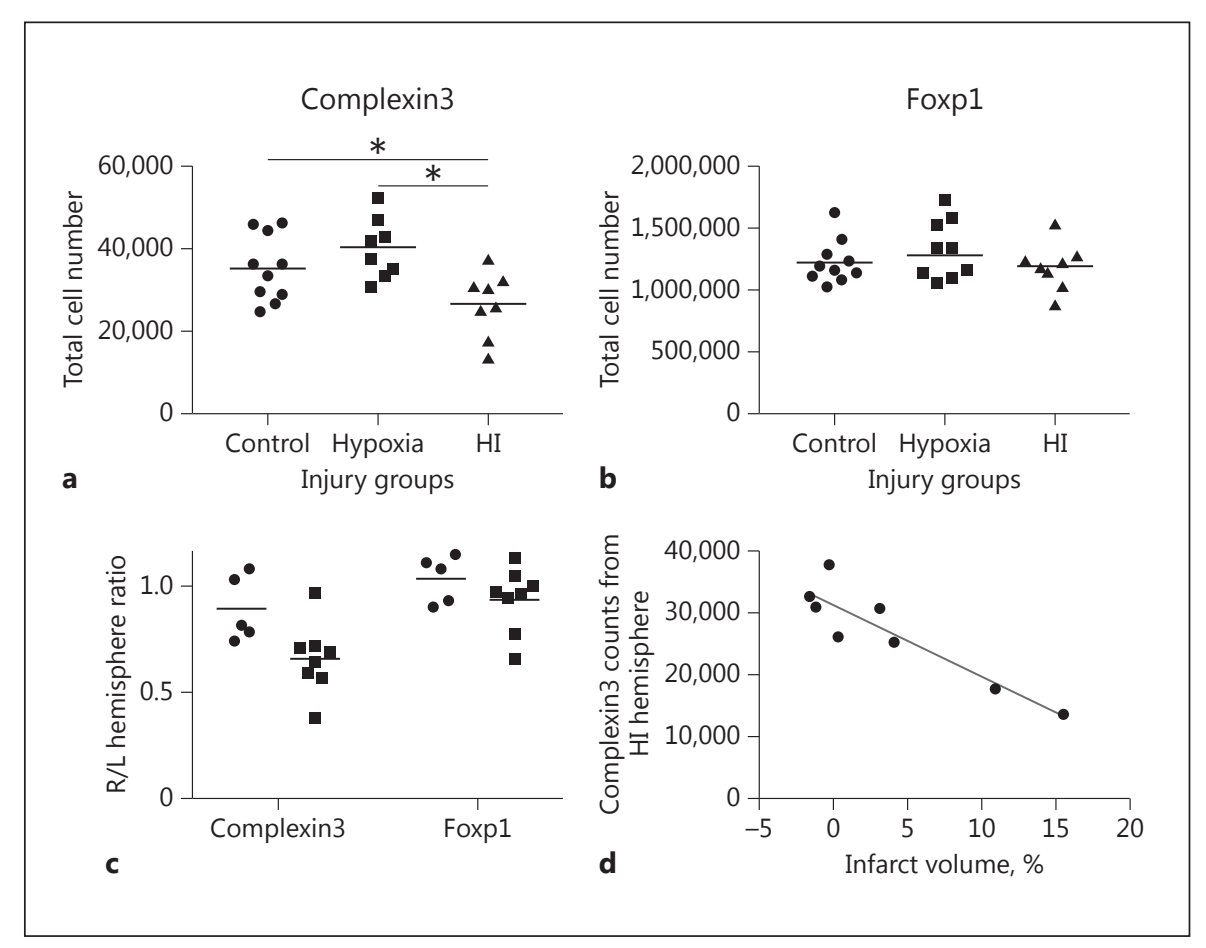

$(n=5)$ (Fig. 4b). We found no significant changes in layer III-IV cell populations across the 3 injury groups. The mean number of Foxp $1+$ cells counted was 1,234,640 \pm 178,540 in the control hemispheres versus $1,195,085 \pm$ 436,609 in the HI hemispheres $(p=0.7)$.

To examine for late decreases in lower-layer populations, we also quantified Foxp1+ cells in the ipsilateral and contralateral hemispheres of moderately injured HI brains $(n=7)$ and compared these with naive controls $(n=8)$ at P21. Foxp1+ cell numbers were not significantly different between injury groups (Fig. 5a). The mean number of Foxp1+ cells counted in the control hemispheres was $1,195,632 \pm 26,912$ versus $1,265,190 \pm 48,089$ in the HI hemispheres $(p=0.19)$. Hemispheric ratios (right/left) of Foxp1 counts had a low variability between control and hypoxia (Fig. 5b). Foxp1 counts were not different when performed at P8 compared with P21, despite a $50 \%$ increase in brain size. HI hemisphere counts at P8 versus P21: $1,195,085 \pm 67,544$ versus $1,265,190 \pm 48,089$ $(p=0.42)$. Control hemisphere counts at P8 versus $\mathrm{P} 21$ : $1,234,640 \pm 56,459$ versus $1,195,632 \pm 26,912(p=0.49)$.

We performed a power analysis to determine the chance of a type II error for the Foxp1+ cortical neuron counts at P8. Using SD from the control sample, the sample size had $92 \%$ power to detect a similar effect size (25\% decrease) to that seen in the Complexin3+ subplate neurons.

Stereological Study of Subplate Neuron Loss after Neonatal HI

\section{Subplate Cell Reduction and Infarct Volumes}

Using birth-dating techniques to identify subplate neurons, we found reduced subplate numbers even in the mild-injury category [6]. Four HI-treated animals in the sample had no change in interhemispheric volume (Fig. 4d), despite a moderate histological grade of injury. Given the arbitrary nature of categorical injury scoring, we sought to determine if subplate neuron loss could be predicted by injury measured by infarct volume. Infarct volumes were closely correlated with the total HI Complexin $3+$ subplate cell number (Fig. $4 \mathrm{~d}, r^{2}=0.82, p=$ $0.0017)$ at P8, but not with the Foxp1+ cell number (Fig. $5 c, r^{2}=0.56, p=0.14$ ) at P21.

\section{Biological versus Stereological Variability in Subplate Neuron Cell Number}

Complexin3-expressing subplate neuron counts demonstrate considerable variability (Fig. $4 \mathrm{a}, \mathrm{CV}$, mean $/ \mathrm{SD}=$ $22 \%$ control Complexin3). To evaluate the sources of variability in our study, we calculated the biological versus stereological variability attributable to our sampling. Observed variance of the group $\left(\mathrm{OCV}^{2}\right.$ group $)$ was made up of true biological variance $\left(\mathrm{CV}^{2}\right.$ biological $)$ and stereological noise $\left(\mathrm{OCE}^{2}\right.$ (individual) $)$ [28]. Biological variance cannot be directly calculated, but observed variance $\left(\mathrm{SD}^{2} / \mathrm{mean}^{2}\right)$ and stereological noise (mean of $\mathrm{CE}^{2}$ ) can. We used the 


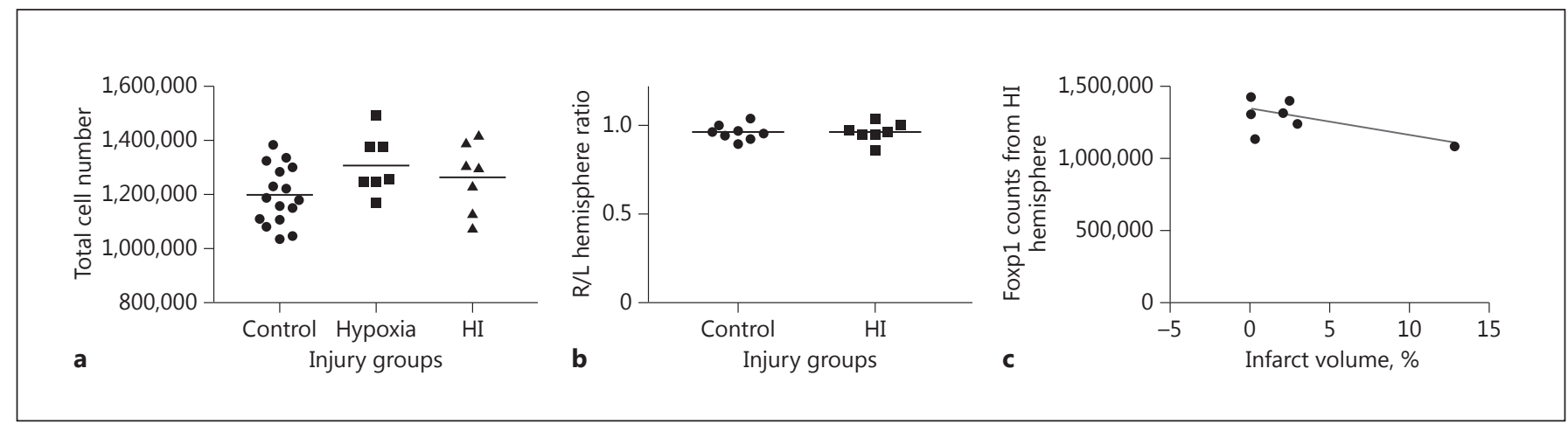

Fig. 5. Quantification of Foxp1 across injury groups at P21. a Foxp $1+$ cell numbers in the different injury groups are not significantly different. b Hemispheric ratios (right/left, R/L) of Foxp1 counts have low variability between control and hypoxia. c Foxp1 cell counts in hypoxia-ischemia (HI) hemisphere are not significantly correlated with infarct volume, as defined by (infarct volume $=[$ hypoxia $-\mathrm{HI}] /$ hypoxia $) . r^{2}=0.56, p=0.1418$.
Gundersen CE, with $m=1$, because data are not "smooth," i.e., there is an inherent difference in the distribution of cell numbers from the front to the back of the brain. While the HI group had twice as much observed variance as the controls ( 0.088 vs. 0.046$)$, both were made up of mostly biological variability ( 89 and $88 \%$, respectively). These values indicate that stereological parameters were optimized for the population sampled, and variability in cell population estimates could be traced back to biological variability and not sampling errors.

\section{Discussion}

We used modern cortical layer-specific markers and definitive counting methods to evaluate subplate neuron loss in comparison to middle/lower-layer cortical neuron loss following early $\mathrm{HI}$ in a rodent model. We found a significant loss of subplate-layer, but not middle/lowerlayer cortical neurons in animals with a moderate degree of brain injury. Moreover, when we examined hemispheric infarct volume as a continuous measurement of brain injury severity, we saw a linear relationship between infarct volume and subplate neuron number.

Okusa et al. [29] examined a similar model but were unable to confirm the specific vulnerability of subplate neurons. As with most reports using this model, they observed significant variability in brain injury severity following $\mathrm{HI}$ at P2. Although they reported only finding strong effects on subplate neurons in severe cases, the images of individual cases labeled with subplate-specific markers show a clear reduction in the moderate cases. A number of factors may have contributed to these different conclusions including experimental and quantification methods, variability inherent in the model, and the evaluation of injury severity. Regarding the quantification methods, the study of Okusa et al. [29] was notable for its large sample size $(n=35)$ and the blinding during quantification. However, the methods include a number of potential sources of bias. First, subplate neuron cell density ratios are analyzed rather than cell numbers, and the individual sample sizes for each injury category are not reported, precluding any consideration of group variability and sample size. Second, sampling within each brain is limited to a single rostral caudal level and 2 sections. Third, no methods are presented for how individual cells were imaged or counted, precluding any assessment of common sources of bias in counting studies. This is especially important with regard to some of the markers quantified including CTGF, which we contend is not suitable for precise cell counting owing to its marker-staining characteristics (i.e., diffuse and irregular profiles). The authors remark on considerable variability in their study and that the standard error of the means for percent cell reduction exceed percent differences many-fold for all markers and all injury categories less than severe. However, they estimate a $14 \%$ (nonsignificant) decrease in Complexin3-expressing subplate neuron cell density in the moderate cases. This effect size is comparable to our results where we noted a $24-34 \%$ reduction between the hypoxia and $\mathrm{HI}$ hemispheres in the moderate cases.

Several factors relating to the methods and inherent variability in the Vannucci model should be considered. First, strain-dependent variability has been well-docu- 
mented in mice [35] and likely exists in rats. SpragueDawley rats were used in the study by Okusa et al. [29]; since our first publication [6], we have exclusively used Long-Evans rats [7,22]. This choice is driven by a need to avoid albino strains for studies on the visual system. Sizonenko et al. [32, 33, 36] report results using the Wistar strain. Differences in the sensitivity of the strains can be noted by differences in the hypoxia dose and duration needed to produce injury: $5.6 \%$ oxygen for $2-3 \mathrm{~h}$ for Long-Evans [7, 22], 6\% oxygen for $2 \mathrm{~h}$ [29] for SpragueDawley, and 6\% oxygen for $30 \mathrm{~min}$ [32] for Wistar. Temperature is also critically important. We maintain our chambers at $34-35^{\circ} \mathrm{C}$ and monitor skin surface temperature, keeping a target temperature of $35.5-37^{\circ} \mathrm{C}$. In the studies by Okusa et al. [29] and Sizonenko et al. [32, 33, 36], chamber temperatures were set to $37^{\circ} \mathrm{C}$. Despite these differences, comparable injury patterns were produced by all the groups (i.e., injury predominantly in the lower layers and cyst formation laterally in more severe cases). What is likely to vary is the distribution of injury, both across strains and with higher temperatures. Despite controlling all these factors, we found considerable variability in the distribution of mild, moderate, and severe injuries across litters. A final methodologic difference relates to the technique of carotid ligation. Okusa et al. [29] ligated and divided the carotid artery, resulting in permanent loss of blood flow. We and other study groups electrocoagulate the carotid artery, a method that produces reversible ischemia with recannulation of the blood flow [37]. Variability of injury severity is a hallmark of hypoxic ischemic brain injury in human newborns.

Variable distribution of injury makes the assessment of severity critical for comparing results across studies. We have reported the histological criteria for our categorical injury grade in great detail and have correlated these histological patterns with diffusion-weighted MRI at $24 \mathrm{~h}$ after injury [7]. In practice, however, histological patterns may vary enough that a categorical injury grade is an artificial construct that is difficult to reproduce across studies and groups. For example, Brockman et al. [34] reported histological patterns and MRI findings very similar to our results, but they included cases in their moderate group that we would grade as severe. Furthermore, in our study, although we selected 8 animals with moderate injury on histology, 4 of them had no measurable difference in hemispheric volume, suggesting a milder injury. Of these 4,3 showed substantial reductions in subplate neuron numbers. In addition to infarct volume, we analyzed cortical thickness as a continuous measure of injury [7]. Cortical thickness predicts alterations in ocular domi-

Stereological Study of Subplate Neuron Loss after Neonatal HI nance plasticity and differences in inhibitory neuron density as well as injury category [7]. However, cortical thickness is extremely dependent upon age and anatomical region. Hemispheric volumes are reliable and reproducible in this model when measured using accepted stereological methods (e.g., the Cavalieri method [22]). Calculation of infarct volumes (contralateral - ipsilateral/contralateral) partially mitigates variability due to brain growth over time in immature rodent models. Considering all these factors, we conclude that infarct volume is the most reliable and unbiased method for measuring injury severity.

In addition to the variability in the Vannucci model itself, we found substantial biological variability in hemispheric subplate neuron numbers $(\mathrm{CV}=22 \%)$. This variability could have arisen from true biologic variability or to stereological noise. A distinct advantage of the Optical Fractionator method is that it allows for the partition of relative-sources variability. Consistent with our low coefficients of error, we found that $>80 \%$ of the variability in subplate neuron numbers came from true biologic variability. In other words, our results would not have been improved substantially by sampling more sections or sites [28]. With the Vannucci procedure, it is common to use the hypoxia-alone hemisphere in comparison to the HI hemisphere, in order to reduce overall experimental variability; this was also done in the study by Okusa et al. [29]. In our study, doing so weakened the relationship between infarct volume and subplate neuron number. While hypoxia exposure alone does not result in overt histological injury or cell death, the treatment does lead to a variety of perturbations at the cellular and signaling level (e.g., the stabilization of HIF1a and HIF signaling).

To the best of our knowledge, this is the first stereological study of subplate neuron number using layer-specific markers. However, Robertson et al. [38] reported results quantifying the ethidium-bromide-stained subplate neuron cell number, purportedly using stereological methods. Their estimates of total subplate neuron number markedly exceeded ours. However, their results must be considered with caution as their methods, described in separate locations as Optical Dissector or Physical Dissector, were actually an amalgamation of both, with elements of $\mathrm{N} \mathrm{v} \times \mathrm{V}$ ref but lacking adequate systematic random sampling. They performed top-counting by comparing 2 confocal image planes, but only sampled a $1-\mathrm{mm}$ linear region. Furthermore, the counts were performed with an automated software program and not using a standard stereological counting frame and rules. No specific markers were used for subplate neurons, and they

Dev Neurosci 2017;39:171-181

DOI: $10.1159 / 000460815$ 
purport to distinguish between neurons and glia based on ethidium-stained nuclear size. Finally, they estimated the subplate-layer volume simply by measuring the brain anterior-posterior and medial-lateral dimensions and assumed an oval shape. While their estimates of the subplate neuron number at P10 exceeded ours at P8 by $\geq 5$ fold, the differences in the methods preclude a direct comparison.

The present study has a number of important limitations. First, the subplate layer is heterogeneous, with multiple subpopulations [25]. By quantifying Complexin3, we likely only captured about $50 \%$ of all subplate neurons [25]. Furthermore, by quantifying the subplate neuron cell number at P8, we underestimated subplate neuron cell loss due to substantial naturally occurring subplate programmed cell death which peaks during the first postnatal week in rats [39]. We chose this age to enable a direct comparison with the study by Okusa et al. [29]. This age also allowed for sufficient time after injury for the resolution of cell death and early edema. A second major issue is that we compared the subplate neuron numbers to a marker, Foxp1, which identifies cells in layers III-V $[40,41]$. Ideally, we would have chosen to quantify layer VI- and layer V-specific markers separately. However, commercially available antibodies for these markers either did not produce selective staining or were not compatible with stereological counting. By repeating the Foxp 1 counts at P21, we ruled out slow or delayed cortical neuronal death.

Why should subplate neurons manifest selective vulnerability? We considered this issue in studies on the immunopurified subplate neuron gene expression and response to excitotoxicity, and found reduced expression of the GluR2 AMPA receptor and increased expression of metabotropic glutamate receptor 3 [42], both of which could account for an intrinsic cellular sensitivity to glutamate excitotoxicity. An additional hypothesis relates to the early generation and maturation of subplate neurons during cortical development [43]. Subplate neurons are among the first cortical neurons generated. They become morphologically mature, receive functional inputs, and are integrated into transient cortical circuits before other cortical neurons $[11,44]$. In the process, they are exposed to sources of potential excitotoxicity before the other cortical layers. This rationale may explain the relative resistance of middle-to-upper-layer neurons, especially layers II and III, in which horizontal connections are known to develop relatively late [45]. Lower cortical layers are predominantly output layers, with descending, callosal, and interareal projections. However, lower cortical layers make and receive excitatory inputs from the subplate and the thalamus [46]. Thus, the early sensitivity of subplate neurons may be a developmental phenomenon and only relative, especially as cortical circuits mature. Lower-layer neurons are also involved with more severe injuries. That being said, subplate neuron injury has a substantial impact on early cortical activity and the activity-dependent neuronal maturation of both excitatory and inhibitory neurons, and it leads to a reduction of multiple forms of cortical plasticity $[7,18,19,21,22]$. All of these effects may have important implications for subsequent cortical function, cognition, and behavior in human infants with preterm brain injury.

\section{Acknowledgments}

This work was supported by National Institutes of Health Grant 5R01NS060896. We thank Donna Ferriero, Fernando Gonzalez, Mark West, and Daniel Friedman for reviewing the manuscript and Amara Larpthaveesarp for helping with the figures.

\section{References}

1 Blencowe $\mathrm{H}$, Cousens $\mathrm{S}$, Oestergaard MZ, Chou D, Moller A-B, Narwal R, et al: National, regional, and worldwide estimates of preterm birth rates in the year 2010 with time trends since 1990 for selected countries: a systematic analysis and implications. Lancet 2012;379:2162-2172.

2 The global burden of preterm birth. Lancet 2009;374: 1214 .

3 Moore T, Hennessy EM, Myles J, Johnson SJ, Draper ES, Costeloe KL, et al: Neurological and developmental outcome in extremely preterm children born in England in 1995 and 2006: the EPICure studies. BMJ 2012;345:e7961.
4 Volpe JJ: Neurology of the Newborn, ed 5. Philadelphia, Saunders/Elsevier, 2008.

5 Ferriero DM: Neonatal brain injury. N Engl J Med 2004;351:1985-1995.

6 McQuillen PS, Sheldon RA, Shatz CJ, Ferriero DM: Selective vulnerability of subplate neurons after early neonatal hypoxia-ischemia. J Neurosci 2003;23:3308-3315.

7 Failor S, Nguyen V, Darcy DP, Cang J, Wendland MF, Stryker MP, et al: Neonatal cerebral hypoxia-ischemia impairs plasticity in rat visual cortex. J Neurosci 2010;30:81-92.
8 Back SA, Han BH, Luo NL, Chricton CA, Xanthoudakis S, Tam J, et al: Selective vulnerability of late oligodendrocyte progenitors to hypoxia-ischemia. J Neurosci 2002;22:455463.

9 Sen E, Levison SW: Astrocytes and developmental white matter disorders. Ment Retard Dev Disabil Res Rev 2006;12:97-104.

10 Hagberg H, Mallard C, Ferriero DM, Vannucci SJ, Levison SW, Vexler ZS, et al: The role of inflammation in perinatal brain injury. Nat Rev Neurol 2015;11:192-208. 
11 Allendoerfer KL, Shatz CJ: The subplate, a transient neocortical structure: its role in the development of connections between thalamus and cortex. Annu Rev Neurosci 1994;17: 185-218.

12 Kanold PO, Luhmann HJ: The subplate and early cortical circuits. Annu Rev Neurosci 2010;33:23-48

13 Luhmann HJ, Kilb W, Hanganu-Opatz IL: Subplate cells: amplifiers of neuronal activity in the developing cerebral cortex. Front Neuroanat 2009;3:19.

14 McConnell SK, Ghosh A, Shatz CJ: Subplate neurons pioneer the first axon pathway from the cerebral cortex. Science 1989;245:978982.

15 Friauf E, McConnell SK, Shatz CJ: Functional synaptic circuits in the subplate during fetal and early postnatal development of cat visual cortex. J Neurosci 1990;10:2601-2613.

16 Friauf E, Shatz CJ: Changing patterns of synaptic input to subplate and cortical plate during development of visual cortex. J Neurophysiol 1991;66:2059-2071.

17 Finney EM, Stone JR, Shatz CJ: Major glutamatergic projection from subplate into visual cortex during development. J Comp Neurol 1998;398:105-118.

18 Kanold PO, Kara P, Reid RC, Shatz CJ: Role of subplate neurons in functional maturation of visual cortical columns. Science 2003;301: 521-525.

19 Kanold PO, Shatz CJ: Subplate neurons regulate maturation of cortical inhibition and outcome of ocular dominance plasticity. Neuron 2006;51:627-638.

20 Dupont E, Hanganu IL, Kilb W, Hirsch S, Luhmann HJ: Rapid developmental switch in the mechanisms driving early cortical columnar networks. Nature 2006;439:79-83.

21 Tolner EA, Sheikh A, Yukin AY, Kaila K, Kanold PO: Subplate neurons promote spindle bursts and thalamocortical patterning in the neonatal rat somatosensory cortex. J Neurosci 2012;32:692-702.

22 Ranasinghe S, Or G, Wang EY, Ievins A, McLean MA, Niell CM, et al: Reduced cortical activity impairs development and plasticity after neonatal hypoxia ischemia. J Neurosci Off J Soc Neurosci 2015;35:11946-11959.

23 Bayer SA, Altman J: Development of layer I and the subplate in the rat neocortex. Exp Neurol 1990;107:48-62.
24 Lein ES, Hawrylycz MJ, Ao N, Ayres M, Bensinger A, Bernard A, et al: Genome-wide atlas of gene expression in the adult mouse brain. Nature 2007;445:168-176.

25 Hoerder-Suabedissen A, Molnar Z: Molecular diversity of early-born subplate neurons. Cereb Cortex 2012;23:1473-1483.

26 West MJ, Slomianka L, Gundersen HJ: Unbiased stereological estimation of the total number of neurons in the subdivisions of the rat hippocampus using the optical fractionator. Anat Rec 1991;231:482-497.

27 Hulley SB, Cummings SR, Browner WS, Newman TB, Hearst N (eds): Designing Clinical Research: An Epidemiologic Approach. Baltimore, Williams \& Wilkins, 1988.

28 West MJ: Optimizing the sampling scheme for a stereological study: how many individuals, sections, and probes should be used. Cold Spring Harb Protoc 2013;2013:521-532.

29 Okusa C, Oeschger F, Ginet V, Wang W-Z, Hoerder-Suabedissen A, Matsuyama T, et al: Subplate in a rat model of preterm hypoxiaischemia. Ann Clin Transl Neurol 2014;1: 679-691.

30 Rice JE 3rd, Vannucci RC, Brierley JB: The influence of immaturity on hypoxic-ischemic brain damage in the rat. Ann Neurol 1981;9: 131-141.

31 Towfighi J, Mauger D, Vannucci RC, Vannucci SJ: Influence of age on the cerebral lesions in an immature rat model of cerebral hypoxia-ischemia: a light microscopic study. Brain Res Dev Brain Res 1997;100:149-160.

32 Sizonenko SV, Sirimanne E, Mayall Y, Gluckman PD, Inder T, Williams C: Selective cortical alteration after hypoxic-ischemic injury in the very immature rat brain. Pediatr Res 2003; 54:263-269.

33 Sizonenko SV, Kiss JZ, Inder T, Gluckman PD, Williams CE: Distinctive neuropathologic alterations in the deep layers of the parietal cortex after moderate ischemic-hypoxic injury in the P3 immature rat brain. Pediatr Res 2005; $57: 865-872$

34 Brockmann MD, Kukovic M, Schönfeld M, Sedlacik J, Hanganu-Opatz IL: Hypoxia-ischemia disrupts directed interactions within neonatal prefrontal-hippocampal networks. PLoS One 2013;8:e83074.

35 Sheldon RA, Sedik C, Ferriero DM: Strainrelated brain injury in neonatal mice subjected to hypoxia-ischemia. Brain Res 1998;810: 114-122.
36 Sizonenko SV, Camm EJ, Garbow JR, Maier SE, Inder TE, Williams CE, et al: Developmental changes and injury induced disruption of the radial organization of the cortex in the immature rat brain revealed by in vivo diffusion tensor MRI. Cereb Cortex 2007;17: 2609-2617.

37 Segovia KN, McClure M, Moravec M, Luo NL, Wan Y, Gong X, et al: Arrested oligodendrocyte lineage maturation in chronic perinatal white matter injury. Ann Neurol 2008;63: 520-530.

38 Robertson RT, Annis CM, Baratta J, Haraldson S, Ingeman J, Kageyama GH, et al: Do subplate neurons comprise a transient population of cells in developing neocortex of rats? J Comp Neurol 2000;426:632-650.

39 McQuillen PS, DeFreitas MF, Zada G, Shatz CJ: A novel role for p75NTR in subplate growth cone complexity and visual thalamocortical innervation. J Neurosci 2002;22: 3580-3593.

40 Ferland RJ, Cherry TJ, Preware PO, Morrisey EE, Walsh CA: Characterization of Foxp2 and Foxp1 mRNA and protein in the developing and mature brain. J Comp Neurol 2003;460: 266-279.

41 Hisaoka T, Nakamura Y, Senba E, Morikawa Y: The forkhead transcription factors, Foxp 1 and Foxp2, identify different subpopulations of projection neurons in the mouse cerebral cortex. Neuroscience 2010;166:551-563.

42 Nguyen V, McQuillen PS: AMPA and metabotropic excitoxicity explain subplate neuron vulnerability. Neurobiol Dis 2010;37: 195-207.

43 Chun JJ, Shatz CJ: The earliest-generated neurons of the cat cerebral cortex: characterization by MAP2 and neurotransmitter immunohistochemistry during fetal life. J Neurosci 1989;9:1648-1667.

44 Kanold PO: Transient microcircuits formed by subplate neurons and their role in functional development of thalamocortical connections. Neuroreport 2004;15:2149-2153.

45 Olavarria J, Van Sluyters RC: Organization and postnatal development of callosal connections in the visual cortex of the rat. J Comp Neurol 1985;239:1-26.

46 Viswanathan S, Bandyopadhyay S, Kao JP, Kanold PO: Changing microcircuits in the subplate of the developing cortex. J Neurosci 2012;32:1589-1601. 